\title{
Chronic Microaspiration and Frailty: A Geriatric Smoking Gun?
}

\author{
Jonathan T. Stewart, MD; Vandan D. Kamath, MS, CCC-SLP; Alejandro V. Jaen-Vinuales, MD; \\ and Inna Sheyner, MD, CMD
}

\section{Chronic microaspiration and diffuse aspiration bronchiolitis may account for some otherwise unexplained frailty in nursing home patients.}

\author{
Author affiliations are listed at \\ the end of the article. \\ Correspondence: \\ Jonathan Stewart \\ (jonathan.stewart1@va.gov)
}

Fed Pract. 2020;37(8):384-387. doi:10.12788/fp.0022
5 railty is a highly prevalent syndrome in nursing homes, occurring in at least $50 \%$ of patients. ${ }^{1}$ The frailty phenotype has been described by Fried and colleagues as impairment in $\geq 3$ of 5 domains: unintentional weight loss, self-reported exhaustion, muscle weakness, slow gait speed, and low physical activity. By this definition, frailty is highly associated with poor quality of life and mortality. ${ }^{2,3}$

In recent years, there has been evolving evidence of a relationship between frailty and chronic systemic inflammation. ${ }^{4-6}$ Some degree of chronic inflammation is likely inherent to the aging process and increases the risk of frailty (so-called inflammaging) but is seen to a greater degree in many pathologic conditions in nursing homes, including cancer, organ failure, and chronic infection.,6-8

Dysphagia also is highly prevalent in nursing homes, affecting up to $60 \%$ of patients and is a strong predictor of hospital utilization and of mortality. ${ }^{9,10}$ Overt aspiration pneumonitis and pneumonia are perhaps the best studied sequelae, but chronic occult microaspiration also is prevalent in this population. ${ }^{11}$ Just as normal systemic inflammatory changes in aging may increase vulnerability to frailty with additional illness burden, normal aging changes in swallowing function may increase vulnerability to dysphagia and to microaspiration with additional illness burden. ${ }^{12,13}$ In older adults, important risk factors for microaspiration include not only overt dysphagia, dementia, and other neurologic illnesses, but also general debility, weakness, and immobility. ${ }^{14}$

Matsuse and colleagues have described diffuse aspiration bronchiolitis (DAB) in patients with chronic microaspiration. ${ }^{14} \mathrm{DAB}$ often goes undiagnosed. ${ }^{14-16}$ As in frailty, weight loss and chronic anemia may be seen, and many of these patients are bedridden. ${ }^{14,17}$ Episodes of macroaspiration and overt lobar pneumonia also may occur. ${ }^{14}$ Lung biopsy or autopsy reveals chronic bronchiolar inflammation and sometimes pulmonary fibrosis, but to date there have been no reports suggesting chronic systemic inflammation or elevated proinflammatory cytokines. ${ }^{14,15,17} \mathrm{We}$ present 3 patients with progressive weight loss, functional decline, and frailty in whom chronic microaspiration likely played a significant role.

\section{CASE 1 PRESENTATION}

A 68-year-old man with a 6-year history of rapidly progressive Parkinson disease was admitted to the Haley's Cove Community Living Center (CLC) on the James A. Haley Veterans' Hospital campus in Tampa, Florida for long-term care. The patient's medical history also was significant for bipolar illness and for small cell carcinoma of the lung in sustained remission.

Medications included levodopa/carbidopa $50 \mathrm{mg} / 200 \mathrm{mg} 4$ times daily, entacapone $200 \mathrm{mg} 4$ times daily, lithium carbonate $600 \mathrm{mg}$ every night at bedtime, lamotrigine $150 \mathrm{mg}$ daily, quetiapine $200 \mathrm{mg}$ every night at bedtime, pravastatin $40 \mathrm{mg}$ every night at bedtime, omeprazole $20 \mathrm{mg}$ daily, tamsulosin $0.4 \mathrm{mg}$ every night at bedtime, and aspirin $81 \mathrm{mg}$ daily. He initially did well, but after 6 months the nursing staff began to notice the patient coughing during and after meals. Speech pathology evaluation revealed moderate oropharyngeal dysphagia, and his diet was downgraded to nectar-thickened liquids.

Over the subsequent 10 months, he 
became progressively weaker in physical therapy and more inactive, with about a 20-lb weight loss and mild hypoalbuminemia of $3.0 \mathrm{gm} / \mathrm{dL}$. He had developed 3 episodes of aspiration pneumonia during this period; a repeat swallow evaluation after the last episode revealed worsened dysphagia, and his physician suggested nil per os (NPO) status and an alternative feeding route. His guardian declined placement of a percutaneous endoscopic gastrostomy (PEG) tube, he was transferred to the inpatient hospice unit, and died 2 weeks later. An autopsy was declined.

\section{CASE 2 PRESENTATION}

A 66-year-old man with a medical history of multiple traumatic brain injuries (TBIs) was admitted to the CLC for long-term care. Sequelae of the TBIs included moderate dementia, spastic paraparesis with multiple pressure injuries, a well-controlled seizure disorder, and severe oropharyngeal dysphagia with NPO status and a percutaneous endoscopic gastrostomy (PEG) tube. His medical history included TBIs and hepatitis $C$ virus infection; medications included levetiracetam 1,000 $\mathrm{mg}$ twice daily, lamotrigine $25 \mathrm{mg}$ twice daily, and cholecalciferol 2,000 U daily. He had multiple stage III pressure injuries and an ischial stage IV injury at the time of admission.

His 11-month stay in the CLC was characterized by progressively worsening weakness and inactivity, with a 25-lb weight loss in spite of adequate tube feeding. Serum albumin remained in the 2.0 to $2.5 \mathrm{gm} / \mathrm{dL}$ range, hemoglobin in the 7 to $9 \mathrm{gm} / \mathrm{dL}$ range without any obvious source of anemia. Most of the pressure injuries worsened during his stay in spite of aggressive wound care, and he developed a second stage IV sacral wound. A single C-reactive protein (CRP) level 2 months prior to his death was markedly elevated at $19.5 \mathrm{mg} / \mathrm{dL}$. In spite of maintaining NPO status, he developed 3 episodes of aspiration pneumonia, all of which responded well to treatment. Ultimately, he was found pulseless and apneic and resuscitation was unsuccessful. An autopsy revealed purulent material in the small airways.

\section{CASE 3 PRESENTATION}

A 65-year-old man with a long history of paranoid schizophrenia and severe gastroesoph- ageal reflux disease had resided in the CLC for about 10 years. Medications included risperidone microspheres $37.5 \mathrm{mg}$ every 2 weeks, valproic acid $500 \mathrm{mg} 3$ times daily and 1,000 mg every night at bedtime, lansoprazole $30 \mathrm{mg}$ twice daily, ranitidine $150 \mathrm{mg}$ every night at bedtime, sucralfate $1,000 \mathrm{mg} 3$ times daily, simvastatin $20 \mathrm{mg}$ every night at bedtime, and tamsulosin $0.4 \mathrm{mg}$ every night at bedtime. He had done well for many years but developed some drooling and a modest resting tremor (but no other signs of pseudoparkinsonism) about 8 years after admission.

There had been no changes to his risperidone dosage. He also lost about $20 \mathrm{lb}$ over a period of 1 year and became increasingly weak and dependent in gait, serum albumin dropped as low as $1.6 \mathrm{gm} / \mathrm{dL}$, hemoglobin dropped to the 7 to $8 \mathrm{gm} / \mathrm{dL}$ range (without any other obvious source of anemia), and he developed a gradually worsening right-sided pleural effusion. CRP was chronically elevated at this point, in the 6 to $15 \mathrm{mg} / \mathrm{dL}$ range and as high as $17.2 \mathrm{mg} / \mathrm{dL}$. Ultimately, he developed 3 episodes of aspiration pneumonia over a period of 2 months. Swallowing evaluation at that time revealed severe oropharyngeal dysphagia and a PEG tube was placed. Due to concerns for possible antipsychotic-induced dysphagia, risperidone was discontinued, and quetiapine $400 \mathrm{mg}$ a day was substituted. He did well over the subsequent year with no further pneumonia and advancement back to a regular diet. He regained all of the lost weight and began independent ambulation. Albumin improved to the $3 \mathrm{gm} / \mathrm{dL}$ range, hemoglobin to the 12 to $13 \mathrm{gm} / \mathrm{dL}$ range, and CRP had decreased to $0.7 \mathrm{mg} / \mathrm{dL}$. The pleural effusion (believed to have been a parapneumonic effusion) had resolved.

\section{DISCUSSION}

All 3 patients met the Fried criteria for frailty, although there were several confounding issues. ${ }^{2}$ All 3 patients lost between 20 and $25 \mathrm{lb}$; all had clearly become weaker according to nursing and rehabilitation staff (although none were formally assessed for grip strength); and all had clear declines in their activity level. Patient 3 had a clear decrement in gait speed, 
but patient 1 had severe gait impairment due to Parkinson disease (although his gait in therapy had clearly worsened). Patient 2 was paraparetic and unable to ambulate. There also was evidence of limited biomarkers of systemic inflammation; all 3 patients' albumin had decreased, and patients 2 and 3 had significant decrease in hemoglobin; but these commonplace clinical biomarkers are obviously multifactorially determined. We have limited data on our patients' CRP levels; serial levels would have been more specific for systemic inflammation but were infrequently performed on the patients.

Multimorbidity and medical complexity are more the rule than the exception in frail geriatric patients, and it is difficult to separate the role of microaspiration from other confounding conditions that might have contributed to these patients' evolving systemic inflammation and frailty. ${ }^{18}$ It might be argued that the decline for patient 1 was related to the underlying Parkinson disease (a progressive neurologic illness in which systemic inflammation has been reported), or that the decline of patient 2 was related to the worsening pressure injuries rather than to covert microaspiration. ${ }^{19}$ However, the TBIs for patient 2 and the schizophrenia for patient 3 would not be expected to be associated with frailty or with systemic inflammation. Furthermore, the frailty symptoms of patient 3 and inflammatory biomarkers improved after the risperidone, which was likely responsible for his microaspiration, was discontinued. All 3 patients were at risk for oropharyngeal dysphagia (antipsychotic medication is clearly associated with dysphagia ${ }^{20}$ ); patient 2 demonstrated pathologic evidence of $\mathrm{DAB}$ at autopsy.

There is evolving evidence that chronic systemic inflammation and immune activation are key mechanisms in the pathogenesis of frailty. ${ }^{4-6}$ It is known that elevated serum levels of proinflammatory cytokines, including tumor necrosis factor- $\alpha$, interleukin- 6 , and CRP are directly associated with frailty and are inversely associated with levels of albumin, hemoglobin, insulin-like growth factor- 1 , and several micronutrients in frail individuals. ${ }^{4-7,21,22}$ Chronic inflammation contributes to the pathophysiology of frailty through detrimental effects on a broad range of systems, including the musculoskeletal, endocrine, and hematopoietic systems and through nutritional dysregulation. ${ }^{2,4,23}$ These changes may lead to further deleterious effects, creating a downward spiral of worsening frailty. For example, it seems likely that our patients' progressive weakness further compromised airway protection, creating a vicious cycle of worsening microaspiration and chronic inflammation.

\section{CONCLUSIONS}

To date, the role of chronic microaspiration and $\mathrm{DAB}$ in chronic systemic inflammation or in frailty has not been explored. Given the prevalence of microaspiration in nursing home residents and the devastating consequences of frailty, though, this seems to be a crucial area of investigation. It is equally crucial for long-term care staff, both providers and nursing staff, to have a heightened awareness of covert microaspiration and a low threshold for referral to speech pathology for further investigation. Staff also should be aware of the utility of the Fried criteria to improve identification of frailty in general. It is probable that covert microaspiration will prove to be an important part of the differential diagnosis of frailty.

\section{Author affiliations}

Jonathan Stewart is a Staff Geropsychiatrist, Vandan Kamath is a Staff Speech and Language Pathologist, Alejandro V. Jaen-Vinuales is a Staff Geriatrician, and Inna Sheyner is the Medical Director, Community Living Center, all at the James A. Haley Veterans' Hospital in Tampa, Florida. Jonathan Stewart is a Professor in Psychiatry and Geriatric Medicine, Alejandro Jaen-Vinuales is an Assistant Professor in Geriatric Medicine, and Inna Sheyner is an Associate Professor in Geriatric Medicine, all at the University of South Florida College of Medicine in Tampa.

\section{Author disclosures}

The authors report no actual or potential conflicts of interest with regard to this article.

\section{Disclaimer}

The opinions expressed herein are those of the authors and do not necessarily reflect those of Federal Practitioner, Frontline Medical Communications Inc., the US Government, or any of its agencies. This article may discuss unlabeled or investigational use of certain drugs. Please review the complete prescribing information for specific drugs or drug combinations-including indications, contraindications, warnings, and adverse effects-before administering pharmacologic therapy to patients.

\section{References}

1. Kojima G. Prevalence of frailty in nursing homes: a systematic review and meta-analysis. J Am Med Dir Assoc. 2015;16(11):940-945. doi:10.1016/j.jamda.2015.06.025

2. Fried LP, Tangen CM, Walston J, et al. Frailty in 
older adults: evidence for a phenotype. J Gerontol A Biol Sci Med Sci. 2001;56(3):M146-M157. doi:10.1093/gerona/56.3.m146

3. Morley JE, Vellas B, van Kan GA, et al. Frailty consensus: a call to action. J Am Med Dir Assoc. 2013;14(6):392-397. doi:10.1016/j.jamda.2013.03.022

4. Chen X, Mao G, Leng SX. Frailty syndrome: an overview. Clin Interv Aging. 2014;9:433-441. doi:10.2147/CIA.S45300.

5. Soysal P, Stubbs B, Lucato $P$, et al. Inflammation and frailty in the elderly: a systematic review and meta-analysis. Ageing Res Rev. 2016;31:1-8. doi:10.1016/j.arr.2016.08.006

6. Langmann GA, Perera S, Ferchak MA, Nace DA, Resnick NM, Greenspan SL. Inflammatory markers and frailty in long-term care residents. J Am Geriatr Soc. 2017;65(8):1777-1783. doi:10.1111/jgs.14876

7. Michaud M, Balardy L, Moulis G, et al. Proinflammatory cytokines, aging, and age-related diseases. J Am Med Dir Assoc. 2013;14(12):877-882. doi:10.1016/j.jamda.2013.05.009

8. Fougere B, Boulanger E, Nourhashemi F, Guyonnet S, Cesari M. Chronic inflammation: accelerator of biological aging. J Gerontol A Biol Sci Med Sci. 2017;72(9):12181225. doi:10.1093/gerona/glw240

9. Shanley C, O'Loughlin G. Dysphagia among nursing home residents: an assessment and management protocol. J Gerontol Nurs. 2000;26(8):35-48. doi:10.3928/0098-9134-20000801-09

10. Altman KW, Yu GP, Schaefer SD. Consequences of dysphagia in the hospitalized patient: impact on prognosis and hospital resources. Arch Otolaryngol Head Neck Surg. 2010;136(8):784-789. doi:10.1001/archoto.2010.129

11. Sakai $\mathrm{K}$, Hirano $\mathrm{H}$, Watanabe $\mathrm{Y}$, et al. An examination of factors related to aspiration and silent aspiration in older adults requiring long-term care in rural Japan. $J$ Oral Rehabil. 2016;43(2):103-110. doi:10.1111/joor.12349

12. Nilsson H, Ekberg O, Olsson R, Hindfelt B. Quantitative aspects of swallowing in an elderly nondysphagic population. Dysphagia. 1996;11(3):180-184. doi:10.1007/BF00366381

13. Daggett A, Logemann J, Rademaker A, Pauloski B. Laryngeal penetration during deglutition in normal sub- jects of various ages. Dysphagia. 2006;21(4):270-274. doi:10.1007/s00455-006-9051-6

14. Matsuse T, Oka T, Kida K, Fukuchi Y. Importance of diffuse aspiration bronchiolitis caused by chronic occult aspiration in the elderly. Chest. 1996;110(5):1289-1293. doi:10.1378/chest.110.5.1289

15. Cardasis JJ, MacMahon H, Husain AN. The spectrum of lung disease due to chronic occult aspiration. Ann Am Thorac Soc. 2014;11(6):865-873. doi:10.1513/AnnalsATS.201310-3600C

16. Pereira-Silva JL, Silva CIS, Araujo Neto CA, Andrade TL, Muller NL. Chronic pulmonary microaspiration: high-resolution computed tomographic findings in 13 patients. J Thorac Imaging. 2014;29(5):298-303. doi:10.1097/RTI.0000000000000091

17. Hu X, Lee JS, Pianosi PT, Ryu JH. Aspiration-related pulmonary syndromes. Chest. 2015;147(3):815-823. doi:10.1378/chest.14-1049

18. Yarnall AJ, Sayer AA, Clegg A, Rockwood K, Parker S, Hindle JV. New horizons in multimorbidity in older adults. Age Aging. 2017;46(6):882-888. doi:10.1093/ageing/afx150

19. Calabrese V, Santoro A, Monti D, et al. Aging and Parkinson's disease: inflammaging, neuroinflammation and biological remodeling as key factors in pathogenesis. Free Radic Biol Med. 2018;115:80-91. doi:10.1016/j.freeradbiomed.2017.10.379

20. Kulkarni DP, Kamath VD, Stewart JT. Swallowing disorders in schizophrenia. Dysphagia. 2017;32(4):467-471. doi:10.1007/s00455-017-9802-6

21. Velissaris D, Pantzaris N, Koniari I, et al. C-reactive protein and frailty in the elderly: a literature review. J Clin Med Res. 2017;9(6):461-465. doi:10.14740/jocmr2959w

22. Hubbard RE, O'Mahoney MS, Savva GM, Calver BL, Woodhouse KW. Inflammation and frailty measures in older people. J Cell Mol Med. 2009;13(9B):3103-3109. doi:10.1111/j.1582-4934.2009.00733.x

23. Argiles JM, Busquets S, Stemmler B, Lotez-Soriano FJ. Cachexia and sarcopenia: mechanisms and potential targets for intervention. Curr Opin Pharmacol. 2015;22:100106. doi:10.1016/j.coph.2015.04.003 